\title{
Early-Infantile Galactosialidosis: Clinical and Radiological Findings
}

Keywords: Galactosialidosis; Non-immune hydrops; Dysostosis; Neonatal ascites

\begin{abstract}
Galactosia lidosis is a rare lysosomal storage disorder. Very few reports of the early-infantile (EIGS) form can be found in the literature. This form typically presents with prenatal non-immune fetal hydrops associated with various complex postnatal clinical findings. We discuss here a case of EIGS seen in our center due to prenatal diagnosis of non-immune hydrops with significant abdominal ascites. Postnatally, the patient was found to have persistent ascites, multiple congenital anomalies, cholestasis, and thrombocytopenia. Radiologic findings suggested osteochondrodysplasia, which in association with the other clinical findings indicated the possibility of a lysosomal storage disorder, such as early-infantile galactosialidosis. We review and discuss here the clinical and radiographic findings in EIGS as described in the literature and compare them to our case study. The information presented should raise awareness of the unusual presentation of early-infantile galactosia lidosis and a id neonatolog ists in the early recognition of this rare and devastating disorder.
\end{abstract}

\section{Introduction}

Galactosialidosis is an autosomal recessive lysosomal storage disease associated with a combined deficiency of enzymes neuraminidase 1 (NEU1) and $\beta$-galactosidase ( $\beta$-GAL). The prevalence of galactosialidosis is unknown; more than 100 cases have been reported with the majority consisting of the juvenile and adult forms. The early infantile form is the most severe and has been reported in 35 cases. These patients have been diagnosed between birth and three months of age with typical findings of nonimmune fetal hydrops, coarse facies, neonatal edema, proteinuria, inguinal hernias and telangiectasias [1]. Hydrops fetalis is typically identifiedon prenatal ultrasound, along with signs of fetal edema and visceromegaly. The skeletal abnormalities previously documented in EIGS are dysostosis multiplex, stippled epiphyses, osteoporosis with coarse trabeculation and significant periosteal reactions [2].

The molecular etiology of EIGS has been described as mutations that occur in the CTSA gene, which codes for a multifunctional lysosomal enzyme protective protein/cathepsin A (PPCA). Loss of function of this gene results in secondary deficiency of $\beta$-GAL and NEU1, which is the biochemical hallmark of the disease [3]. There are remarkable similarities between EIGS and congential sialidosis (an isolated deficiency in NEU1), including fetal hydrops, ascites, visceromegaly, and early death. Therefore it is well understood the key role NEU1 deficiency plays in the pathogenesis of GS. It is not as clear how cathepsin $A$ and $\beta-G A L$ deficiency contribute to the phenotypic abnormalities of the disease and further explanation is pending [1].

\section{Case Report}

We report a newborn patient born to a 23 year old G3P0 mother and a 26 year old father, both of Indian (Gujurat) descent at 30

\section{Journal of}

Clinical \& Medical Case Reports

Callyn B. Riggs ${ }^{1}$, Luis F. Escobar ${ }^{2 *}$, and Megan Tucker $^{2}$

${ }^{1}$ Department of Pediatrics, Peyton Manning Children's Hospital, Indianapolis, IN 46260, USA

${ }^{2}$ Medical Genetics \& Neurodevelopmental Center, Peyton Manning Children's Hospital, Indianapolis, IN 46260, USA

*Address for Correspondence:

Luis F. Escobar, MD, Medical Genetics \& Neurodevelopmental Center, Peyton Manning Children's Hospital, 8402 Harcourt Rd, \#300, Indianapolis, IN 46260, USA, Tel: 317-338-5288; Fax: 317338-7154; E-mail: LFEscoba@stvincent.org

Submission: 19 August 2015

Accepted: 29 October 2015

Published: 03 November 2015

Copyright: (c) 2015 Riggs CB, et al. This is an open access article distributed under the Creative Commons Attribution License, which permits unrestricted use, distribution, and reproduction in any medium, provided the original work is properly cited.

weeks plus 5 days gestational age. There was a history of maternal beta-thalassemia trait, with negative hemoglobin electrophoresis testing for the father. Both parents were otherwise healthy but subsequently found to be likely consanguineous after significant loss of heterozygosity across chromosomes was identified by SNP array. Family history was negative for birth defects, mental retardation, or known chromosomal disorders. The mother had two previous spontaneous abortions. Pregnancy was complicated by nonimmune hydrops fetalis, and prenatal ultrasound evidence of echogenic bowel, ventriculomegaly, short bones, significant abdominal ascites, and a 2 -vessel cord. In order to facilitate delivery fetal peritoneal aspiration was performed immediately prior to cesarean section delivery which also help to decrease diaphragmatic and pulmonary compression and allowed improve pulmonary function. Close to $150 \mathrm{ml}$ of amber colored fluid was removed. The infant was delivered emergently via cesarean section due to breech presentation and variable fetal heart rate decelerations with reversed diastolic flow. Apgar scores were 2 at 1 minute, 5 at 5 minutes, and 8 at 10 minutes. Meconium stained fluid was present at delivery. The infant appeared cyanotic with respiratory depression, underwent tracheal suctioning then endotracheal intubation with surfactant administration at 10 minutes of life. He did not require chest compressions during resuscitation. Chest radiograph shortly after birth revealed diminished lung volumes with diffuse granular opacities, thus infant was admitted to the Neonatal Intensive Care Unit and placed on high-frequency oscillatory ventilation (HFOV).

At birth patient's weight was $1.285 \mathrm{~kg}$ ( $20^{\text {th }}$ percentile $), 33 \mathrm{~cm}$ in length $\left(<1^{\text {st }}\right.$ percentile), with a head circumference of $27.5 \mathrm{~cm}$ $\left(23^{\text {rd }}\right.$ percentile). Physical exam revealed triangular facies and head, firm scalp edema, bilateral red reflex, low-set ears, and dysplastic and posteriorly rotated but intact palate. There were coarse breath sounds with marked respiratory distress and retractions, and a normal cardiovascular exam. The abdomen was soft but distended with moderate ascites, hepatomegaly and a left inguinal hernia. 
Citation: Riggs CB, Escobar LF, Tucker ME. Early-Infantile Galactosialidosis: Clinical and Radiological Findings. J Clin Med Case Reports. 2015;2(2): 4.

Genitourinary exam was unremarkable with normal preterm male features, bilateral descended testes and patent anus. The infant was responsive to stimuli with mildly decreased muscle tone. A short neck was noted but no gross vertebral abnormalities, bilateral complete transverse palmar creases were present Rhizomelic shortening of the arms and bilateral shortening of the lower extremities was observed. The patient underwent work up on day one of life including renal ultrasound, which revealed small, echogenic kidneys with decreased corticomedullary differentiation, and echocardiogram, showing a large patent ductus arteriosus, moderate sized perimembranous ventricular septal defect and moderate sized atrial septal defect secundum with left to right shunt. Echoencephalography was also performed with results showing partial absence of the corpus callosum posteriorly, questionable hypoplasia of the cerebellar vermis and small subependymal cysts bilaterally. Skeletal radiographs obtained (Figure 1) showed irregular epiphyseal areas of the long bones with mild bowing, indicating the possibility of bone dysplasia, a skeletal dysplasia panel was sent with normal results. A complete skeletal survey (Figure 2) was performed on day five of life and results of this study showed: 1) Demineralized appearance of the bones, particularly the skull, with an abnormal course and appearance of the bony trabeculae with metaphyseal cuffing and rachitic rosary appearance of the anterior portion of the ribs; 2) Distal right femur metadiaphyseal fracture in near anatomic alignment; 3) Lack of visualization of developing teeth within the maxilla and mandible; 4) The ankle area showed suspected chondrodysplasia. The workup also included a negative molecular hypophosphatasia testing, unremarkable urine for organic acids, negative 10 gene skeletal dysplasia panel, and an unremarkable SNP array with the exception

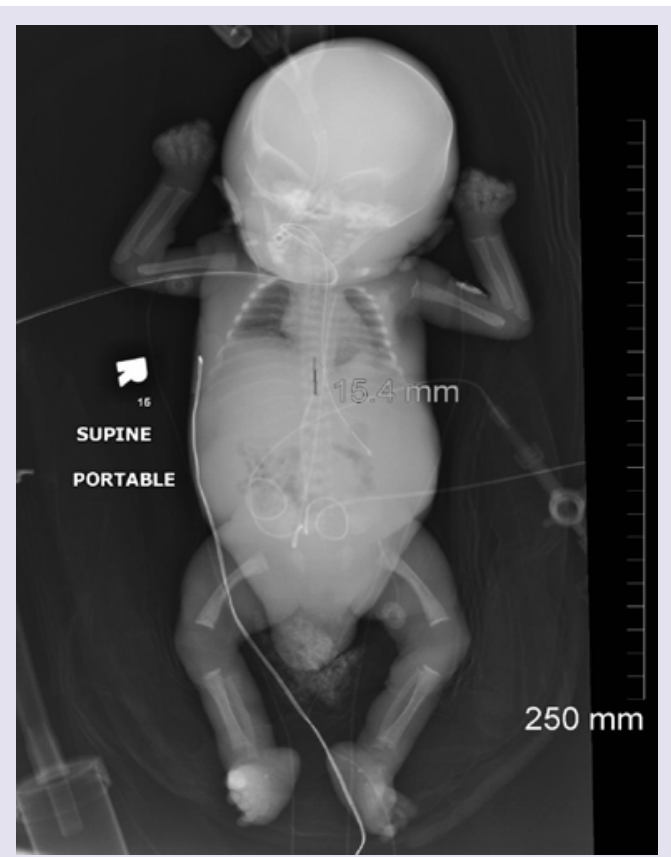

Figure 1: Initial radiograph taken shortly after birth showing the limbs appea disproportionately short compared to the body. The humeri appear equal in length compared to the radius and the femurs appear equal in length relative to the tibias, suggesting rhizomelic skeletal dysplasia. In addition, skeletal features are predominantly those of metaphyseal irregularity, mild bowing of the long-bones, and likely osteopenia. of significant loss of heterozygosity across 8 different chromosomes and involving at least 900 genes including CTSA which is suggestive of possible consanguinity. The lysosomal panel through Greenwood Genetics, LLC showed decreased activity of beta galactosidase and alpha neuraminidase indicating galactosialidosis. This was confirmed by abnormal CTSA sequencing anaylsis indicating a homozygous $833 \mathrm{~T}>\mathrm{C}$ variant, which is expected to be pathogenic.

Additional lab abnormalities found in this case were persistent neutropenia with initial absolute neutrophil count of 605 and remaining below 1000 for his entire course, in addition to anemia and thrombocytopenia requiring multiple blood and platelet transfusions. Urine testing for cytomegalovirus was negative. The patient's hospital course continued to be complicated by respiratory distress with several failed attempts at extubation. He remained on antibiotics since birth due to concerns for infection in the setting of pancytopenia. Significant abdominal ascites persisted with abdominal girth increasing from 23 to $32 \mathrm{~cm}$. Peritoneal aspirations were not performed due to the expectation that fluid would re-accumulate and an aspiration would only provide temporary relief. The decision was made to withdraw life support and the patient died at 38 days of life.

\section{Discussion}

The clinical findings of this case, particularly non-immune hydrops fetalis, suggest the diagnosis of a storage disorder, however it can be difficult to differentiate between the numerous lysosomal storage disorders based on phenotypic findings alone. Other genetic conditions causing non-immune fetal hydrops include Gaucher's disease, generalized gangliosidosis, Salla's disease, sialidosis, mucopolysaccharidosis types IV and VII, and Tay-Sach's disease. Galactosialidosis shares many of these physical findings with other lysosomal storage disorders, such as coarse facies, edema, abnormal bone formation and cherry red spots on eye exam [4]. Visceromegaly is another common finding of storage disorders, however hepatosplenomegaly is more commonly seen in EIGS rather than enlargement of the heart or kidneys. There have been 14 cases of EIGS that report heart involvement, typically cardiomegaly, thickened septum and subsequent cardiac failure [1]. Kidney involvement usually manifests at proteinuria, and kidney enlargement has not been documented in cases of galactosialidosis, but has been reported in cases of glycogen storage disease type I and muchopolysaccharidoses [5].

In EIGS, reports of punctate epiphyses, dysostosis multiplex, osteoporosis with coarse trabeculation, and significant periosteal reactions have made for skeletal findings [2]. Punctate epiphyseal calcifications (PEC) can be seen in several lysosomal storage disorders (particularly $\mathrm{GM}_{1}$ gangliosidosis, sialidosis, and mucopolysaccharidosis) chromosomal anomalies, peroxismal disorders, vitamin $\mathrm{K}$ disorders, toxic conditions, and certain syndromes, such as Smith-Lemli-Opitz syndrome. The pathogenesis for punctate epiphyseal calcifications is not fully understood, but it is considered altered lysosomal metabolism in lysosomal storage diseases accelerate the transformation of rough endoplasmic reticulum and lysosome-rich cells into osteogenic cells that induce calcification. Regardless of the mechanism, the presence of punctate epiphyseal calcifications on radiographic studies should create suspicion for diagnosing lysosomal storage disorders [6]. Skeletal 
Citation: Riggs CB, Escobar LF, Tucker ME. Early-Infantile Galactosialidosis: Clinical and Radiological Findings. J Clin Med Case Reports. 2015;2(2): 4.

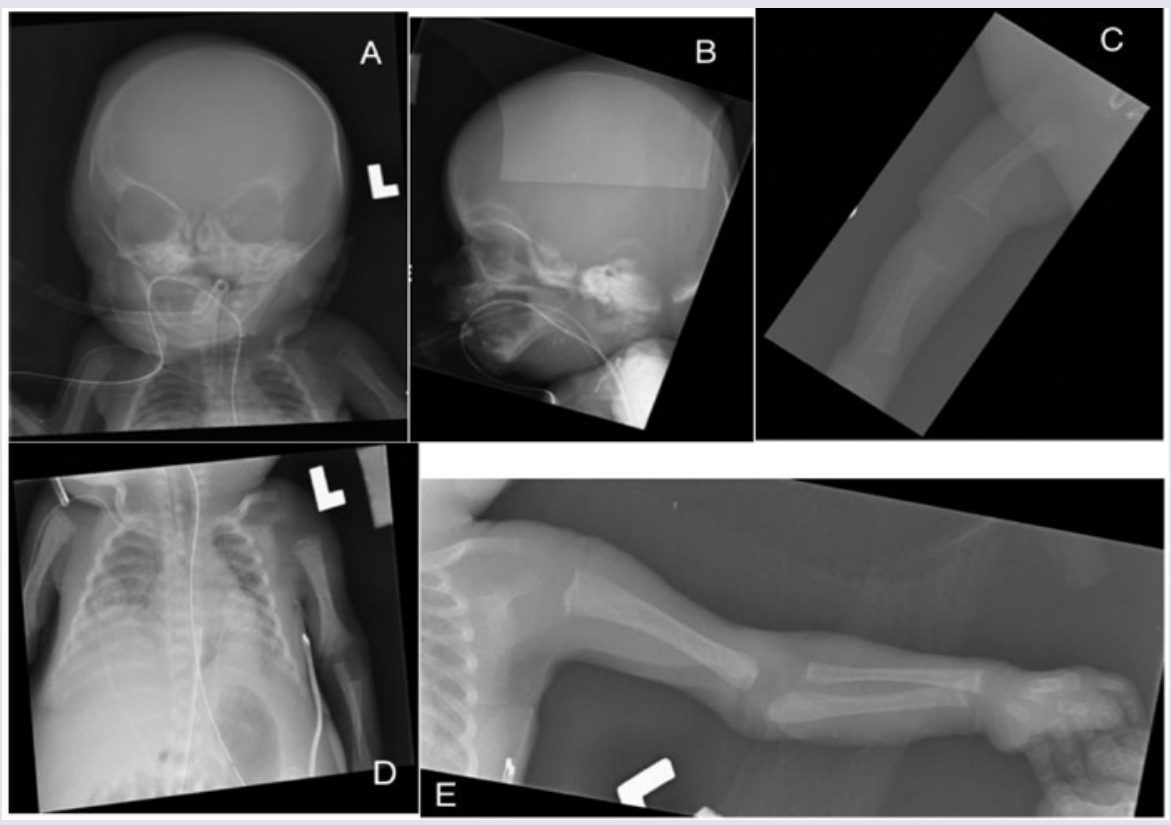

Figure 2: Skeletal survey obtained on day 5 of life. A,B) The skull is hyperlucent throughout indicating a demineralized appearance. C) The long bones demonstrate abnormal course trabeculae with metaphyseal cuffing identified. D) There is a metaphyseal fracture identified in a transverse fashion to the distal right femur. The long bones do not appear dysmorphic other than the abnormal appearance to the bony matrix and metaphyseal cuffing did not appear dysmorphic. E) There is prominence to the anterior ribs consistent with a rachitic rosary type appearance.

Table 1: Clinical features of early infantile galactosialidosis (EIGS).

\begin{tabular}{|l|c|c|}
\hline & $\begin{array}{c}\text { Number of previous } \\
\text { cases with finding } \\
\text { present }\end{array}$ & Present in this patient \\
\hline Total number of patients & 35 & 1 \\
\hline Sex (male/female) & $13 \mathrm{M} / 14 \mathrm{~F}$ & Male \\
\hline Parental consanguinity & 7 & + \\
\hline Age at onset of & $0-90(12)$ days & + \\
\hline symptoms (mean) & 21 & - \\
\hline Coarse facies & 2 & + \\
\hline Macrocephaly & 27 & + \\
\hline Hepatosplenomegaly & 25 & + \\
\hline Fetal hydrops, ascites & 10 & + \\
\hline Kidney involvement & 14 & + \\
\hline Heart involvement & 9 & - \\
\hline Hernias & 1 & - \\
\hline $\begin{array}{l}\text { Anemia and } \\
\text { thrombocytopenia }\end{array}$ & 9 & - \\
\hline Telangiectasias & 1 & - \\
\hline Seizures & 3 & - \\
\hline Cherry-red spots & 1 & days \\
\hline Lens opacity & 5 & + \\
\hline Corneal clouding & 7 & + \\
\hline Dysostosis multiplex & & + \\
\hline Vertebral changes & months/7 months & + \\
\hline $\begin{array}{l}\text { Age at death (range/ } \\
\text { mean) }\end{array}$ & & + \\
\hline Reveiwed & & + \\
\hline
\end{tabular}

${ }^{1}$ Reveiwed in d'Azzo et al., 2013

changes overall seem to be less prominent in the EIGS than the late infantile and juvenile/adult forms of galactosialidosis likely secondary to the short life spans of these patients, which averages 7 months of age. Dysostosis multiplex is most commonly seen in the late infantile form and is rare in the juvenile/adult type [1]. The radiographic finding of bone dysplasia was a key to diagnosis in the case discussed in this paper. Osteochondrodysplasia differs from dysostoses in regards to the defects being intrinsic to the growth of the bone and cartilage, and as a result of gene expression, the abnormalities can evolve to alter previously unaffected bones. Dysostoses causes defective bone formation secondary to defects in blastogenesis in the first 6 weeks of fetal life, thus the abnormalities do not spread to previously uninvolved bones [7].

Based on the information provided here, it is important to highlight clinical findings more specific to galactosialidosis in addition to features not typically seen with this condition in order to help clinicians in early detection and diagnosis. Table 1 highlights the clinical features of EIGS (reviewed in d'Azzo et al., 2013) and indicates which findings were present in the patient described in this case report. Treatment for galactosialidosis is only symptomatic, and EIGS may lead to early demise within the first year of life. Accurate diagnosis is of extreme help to families in general and would aid clinicians in the management of this severe and rare condition. Providing families with genetic counseling and directed prenatal diagnosis with risk evaluation in subsequent pregnancies is essential.

\section{References}

1. d'Azzo A, Andria G, Strisciuglio P, Galjaard H (2001) Galactosialidosis. In Scriver CR, Beaudet AL, Sly WS, Valle D (eds.). The metabolic \& molecular bases of inherited disease. Vol. III (8th edn) New York: McGraw-Hill, pp: 3811.

2. Patel MS, Callahan JW, Zhang S, Chan AK, Unger S, et al. (1999) Earlyinfantile galactosialidosis: prenatal presentation and postnatal follow-up. Am J Med Genet 85: 38-47.

3. Caciotti A, Catarzi S, Tonin R, Lugli L, Perez CR, et al. (2013) Galactosialidosis: review and analysis of CTSA gene mutations. Orphanet J Rare Dis 8: 114. 
Citation: Riggs CB, Escobar LF, Tucker ME. Early-Infantile Galactosialidosis: Clinical and Radiological Findings. J Clin Med Case Reports. 2015;2(2): 4.

ISSN: $2332-4120$

4. Carvalho S, Martins M, Fortuna A, Ramos U, Ramos C, et al. (2009) Galactosialidosis presenting as nonimmue fetal hydrops: a case report. Prenat Diagn 29: 895-896.

5. Schwartz IV, Artigalas O, Ries M, Clarke JT, Giugliani R, et al. (2009) Punctate calcifications in lysosomal storage disorders. Clin Dysmorphol 18: 172-177
6. Offiah AC, Hall CM (2003) Radiological diagnosis of the constitutional disorders of bone. As easy as A, B, C? Pediatr Radiol 33: 153-161.

7. Sewell AC, Pontz BF, Weitzel D, Humburg C (1987) Clinical heterogeneity in infantile galactosialidosis. Eur J Pediatr 146: 528-531. 